Research Article

CrossMark

\&cickfor updates:

\title{
Effect of Varying Dietary Protein Levels on Hematology of Labeo calbasu
}

\author{
Saira Bano, Muhammad Naeem* and Samrah Masud
}

Institute of Pure and Applied Biology, Zoology Division, Bahauddin Zakariya University, Multan-60800, Pakistan

\begin{abstract}
The present work is the first detailed report from Pakistan on study of blood parameters in Labeo calbasu, under three treatments (T1,T2 and T3) fed at different crude protein (CP) levels ratios i.e., 15\%, 20\% and $25 \% \mathrm{CP}$. Fish were first collected, acclimatized, kept in triplicate aquaria under controlled conditions in three groups (T1, T2 and T3) and then 5 fish were selected from each treatment at the end of 12 weeks of feeding trial from January to April, 2018, during which fish were fed at the rate of $4 \%$ of their body weight. The blood variants were studied like Lymphocytes (LYM), Monocytes (MON), Granulocytes (GRA), Red Blood Cells (R.B.C), Hemoglobin (Hgb), Hematocrit (HCT), Mean Corpuscular volume (MCV), Mean Corpuscular Haemoglobin (MCH), Red Blood Cell Distribution Width when measured with Standard Deviation (RDW-SD), Mean Corpuscular Haemoglobin Concentration (MCHC), Platelets (PLT), Plateletcrit (PCT), Mean Platelets Volume (MPV), Platelets Distribution Width (PDW) For statistical analysis, regression was applied to the data. Fish fed with 25\% dietary protein level under T3 showed good health status as compared to T1 and T2 with $15 \%$ and $20 \%$ dietary protein levels. Results of the research work indicated that $L$. calbasu reared by feeding $25 \% \mathrm{CP}$ showed better health status.

Received | September 13, 2019; Accepted | Mach 08, 2020; Published | April 25, 2020

*Correspondence | Muhammad Naeem, Institute of Pure and Applied Biology,Zoology Division, Bahauddin Zakariya University, Multan-60800, Pakistan; Email: dr_naeembzu@yahoo.com

Citation | Bano, S., M. Naeem and S. Masud. 2020. Effect of Varying Dietary Protein Levels on Hematology of Labeo calbasu. Sarhad Journal of Agriculture, 36(2): 507-516.

DOI | http://dx.doi.org/10.17582/journal.sja/2020/36.2.507.516

Keywords | Hematology, Dietary protein levels, Carp, Regression equations, Body size
\end{abstract}

\section{Introduction}

$\mathrm{T}$ he hematological parameters analysis like red blood cells, white blood cells, hematocrit and hemoglobin concentration are commonly useful as predictive and indicative tools in aquaculture to determine both wild and cultured fish fitness and health rank. The parameters have also been used as functional gauges of environmental disorder and pollution (Burgos-Aceves, 2019).

Fish is a sound and nutritious wellspring of nutrition (Naeem et al., 2010). Fish that seems outwardly healthy may not be essentially healthy. The consumers often assess fresh fish by its external look but its nutritive value does not depend on its appearance. Hematology, serum analysis and plasma biochemistry are some virtuous parameters for the evaluation of fish health (Adeshina et al., 2019). The biochemical and hematological profiles of a fish are also helpful instruments in the evaluation of performance, suitability and wellbeing status of cultivated fish and creatures (Iqbal and Naeem, 2016).

The biochemical and hematological qualities might be influenced by a few components viz animal varieties, stress, sex, natural conditions, dietary and physiological status (Moradi et al., 2013; Najim et al., 2014). June 2020 | Volume 36 | Issue 2 | Page 507 
The varieties in blood parameters record modification, because of, variety in quality and making of feed. The RBCs tally demonstrates a noteworthy minor departure from advantageous artificial feeds (Iqbal and Naeem, 2016; Khan and Maqbool, 2017).

According to Nasir and A1-Sraji (2013), the principle capacity of white blood cells is to battle diseases, protection by phagocytosis, generation, transportation and circulation of antibodies in safe reactions. The most prominent job of platelets is to start thickening of blood during the time spent in hemostasis according to Ferdous and Scott (2015).

Khan and Maqbool (2017) found an expansion in HCT, Hgb and RBC estimations encouraged with differing dimensions of dietary protein, appearing general pattern that with the increase of dietary protein levels, $\mathrm{Hb}, \mathrm{HCT}$ and RBC will likewise be expanded.

In the field of research, various reports of the impact of artificial diets on blood parameters of fish are recorded up till now, but more work is needed. To the best of our knowledge, the blood profile of Labeo calbasu fed at different feeding regime is not previously reported in the literature from Pakistan, so its health status can be estimated by the present work on its hematology.

The present study aims to study the effect of three feeding levels of protein on hematological indices to estimate the health status of Labeo calbasu under controlled conditions.

\section{Materials and Methods}

The samples of Labeo calbasu were collected from Head Islam, Tehsil Hasil Pur, District Bahawalpur, Punjab by using hand nets, during the month of January, 2018. The samples were brought in oxygenated plastic bags to fisheries Lab, IPAB, Bahauddin Zakariya University, Multan. Fish were kept in triplicate aquaria under controlled conditions in three different groups of treatments (T1, T2, and T3) on the basis of three different crudes (dietary) protein levels, $15 \%, 20 \%$ and $25 \% \mathrm{CP}$. The fish were acclimatized in aquaria for a period of two weeks during which rice polishing was fed to the fish. After the end of the feeding trial of 12 weeks, 5 fish samples were taken randomly from each of the three treatment aquaria T1, T2 and T3 with $15 \%, 20 \%$ and $25 \%$ dietary protein level respectively for hematological studies.
The live fish were anesthetized with clove oil before taking blood. The rate of use of clove oil for anesthetizing fish was $50 \mu \mathrm{l}$ of clove oil per liter of water just before taking blood. Prior to take blood samples fish were weighed and length of the fish at that time was also determined. The blood samples for haematological studies were drained from caudal peduncle of the fish or by cardio puncturing depending on the fish weight and size as per suitability. The blood samples were collected from the fish by using a disposable plastic syringe (of 23 Gauge), which was already rinsed with EDTA solution $(2.7 \%)$ for analysis of some haematological parameters like $\mathrm{RBC}$ and its indices, WBC and its parameters and platelets and its parameters as well as hemoglobin. The collected blood was immediately transferred to vials containing EDTA (as an anti-coagulant). The collected sample than were analyzed for CBC in the haematological analyzer (model: Medonic M-Series $\mathrm{M}_{32} \mathrm{M}$ ) present in laboratory of IP and AB (Institute of Pure and Applied Biology), BZU Multan. Different blood parameters were determined by the haematological analyzer. Theses parameters were LYM (Lymphocytes), MON (Monocytes), GRA (Granulocytes), R.B.C (Red blood cells), Hgb (Haemoglobin), HCT (Hematocrit), MCV (Mean corpuscular volume), $\mathrm{MCH}$ (Mean corpuscular haemoglobin), RDW (Red blood cell distribution width), MCHC (Mean corpuscular haemoglobin concentration), RDW-SD (Red blood cell distribution width found with standard deviation), PLT (Platelets), PCT (plateletcrit value), MPV (Mean platelet volume) and PDWC (Platelet distribution width concentration).

The data then subjected to statistical analysis. ANOVA was applied at 0.01 significant level. SPSS statistical software version 16 was applied to compare mean values. Regression analyses were also applied to study correlation of fish size (total length and body weight) with haematological parameter following Iqbal and Naeem (2016).

\section{Results and Discussion}

Hematological indices of the experimental fish Labeo calbasu were studied under three different treatments i.e., T1 with $15 \%$, T2 with $20 \%$ and T3 with $25 \%$ dietary protein levels. The hematological parameters indicated the health of Labeo calbasu. The blood profile of fishes is similar to other members of phylum 
Vertebrata in having blood cells i.e., Red blood cells (RBCs), White blood cells (WBCs), platelets and plasma. The plasma contains minerals, water, enzymes, electrolytes and hormones.

Mean values and ranges of various hematological variables like $\mathrm{WBC}$ and its indices, $\mathrm{RBC}$ and its indices, platelets and its indices and hemoglobin etc. as given in Table 1 .

\section{WBCs or white blood cells in $10^{3} / \mu \mathrm{L}$}

Table 1 describes white blood cells count in Labeo calbasu under three different treatments. The mean value with standard deviation in the case of WBCs in treatment $\mathrm{T} 1$ with $15 \%$ dietary protein level was found to be $78.74 \pm 6.82$ and range was $67.5-87.2$ $\left(10^{3} / \mu \mathrm{L}\right)$. The mean value with standard deviation $84.58 \pm 5.55$ and range was 77.5-92.4 in treatment $\mathrm{T} 2$ with $20 \%$ dietary protein level while in case of T3 with $25 \%$ dietary protein level the mean value with standard deviation was $75.58 \pm 4.43$ and the range was 68.9-81.2. As WBCs were comparatively higher in both T1 and T2 showing stressful health conditions of Labeo calbasu in these treatments and low WBCs in T3 showed its good health profile.

\section{Lymphocytes $\left(10^{3} / \mu \mathrm{L}\right)$}

Lymphocytes count in T1 with $15 \%$ dietary protein level having mean $72.56 \pm 6.29$ and range 62.5-81.1. In case the of T2 mean was $76.88 \pm 5.56$ and range 69.684.3.T3 had mean $69.76 \pm 4.32$ and range $63.4-74.6$. The order of arrangement of lymphocytes was T3>T2 $>\mathrm{T} 1$.

\section{Monocytes $\left(10^{3} / \mu L\right)$}

Monocytes count was found in case of T1 mean was $4.44 \pm 0.47$ and range 3.6-4.9. In case of $\mathrm{T} 2$ mean was $5.6 \pm 0.72$ and range 4.3-6.4. In case of T3 mean was $4.1 \pm 0.4$ and range 3.5-4.7. The order of presence of monocytes were $\mathrm{T} 2>\mathrm{T} 1>\mathrm{T} 3$.

\section{Granulocytes $\left(10^{6} / \mu \mathrm{L}\right)$}

In T1 mean was $1.47 \pm 0.53$ and range $1.2-2.7$. In T2 mean was $2.1 \pm 0.87$ and range 1.0-3.6. In T3 mean was $1.72 \pm 0.81$ and range was $0.9-2.7$. The presence was in a sequence $\mathrm{T} 2>\mathrm{T} 3>\mathrm{T} 1$.

\section{Lymphocytes (\%)}

In T1 mean was 92.16 \pm 0.69 and range 91.03-93.00. In T2 mean was 90.86 \pm 0.94 and range 89.81-92.42. In T3 mean was 92.29 1.34 and range 90.33-94.42. In sequence it could be T3>T1>T2.
Monocytes (\%)

In T1 mean was 5.64 \pm 0.31 and range 5.28-6.14. In T2 mean was $6.62 \pm 0.77$ and the range was $5.55-7.7$. In T3 mean was $5.46 \pm 0.72$ and range $4.44-6.24$. The sequence was $\mathrm{T} 2>\mathrm{T} 1>\mathrm{T} 3$.

\section{Granulocytes (\%)}

In T1 mean was $2.21 \pm 0.61$ and the range was 1.50 3.23. In T2 mean was $2.52 \pm 1.16$ and range 1.17-4.65. In T3 mean was $2.25 \pm 0.98$ and the range was $1.14-$ 3.53. The presentation order was $\mathrm{T} 2>\mathrm{T} 3>\mathrm{T} 1$.

\section{Red blood cells $\left(10^{6} / \mu \mathrm{L}\right)$}

In T1 mean was $1.43 \pm .0 .19$ and range was $1.13-1.72$. In T2 was $1.85 \pm 0.16$ and range was $1.65-2.05$. In T3 mean was $1.17 \pm 0.11$ and range $0.99-1.28$. The order of volume of RBC in the blood of $L$. calbasu in different treatments was found as $\mathrm{T} 2>\mathrm{T} 3>\mathrm{T} 1$.

\section{Hemoglobin $(\mathrm{g} / \mathrm{dl})$}

In T1 mean was $7.74 \pm 0.30$ and range was $7.3-8.2$. In T2 mean was $7.66 \pm 0.16$ and range was $7.5-7.9$. In T3 mean was $8.16 \pm 0.31$ and range was 7.6-8.5. $\mathrm{T} 3>\mathrm{T} 1>\mathrm{T} 2$ was a sequence.

\section{Hematocrit (\%)}

In T1 mean was $31.58 \pm 2.54$ and range was 28.6-35.5. In T2 mean was $34.16 \pm 0.96$ and range was 32.6-35.3. In T3 mean and range were $37.32 \pm 1.64$ and 34.639.4 respectively. The arrangement with respect to presence $\mathrm{T} 3>\mathrm{T} 2>\mathrm{T} 1$.

\section{Mean corpuscular volume ( $\left.\mu m^{3}\right)$}

In case of T1 mean was $161.52 \pm 16.10$ and range was 133.4-182.7. In T2 mean was $167.92 \pm 8.09$ and range was 158.0-176.8. In T3 mean was $123.94 \pm 6.28$ and range was $112.7-131.2 . \mathrm{T} 2>\mathrm{T} 1>\mathrm{T} 3$.

\section{$M C H(p g)$}

In case of $\mathrm{T} 1$ mean was $58.32 \pm 5.78$ and range was 52.3-69.2. In $\mathrm{T} 2$ mean was $41.79 \pm 3.66$ and range was 38.05-46.75. In T3 mean and range were 70.44 \pm 5.42 and $63.28-76.77$ respectively. $\mathrm{T} 3>\mathrm{T} 1>\mathrm{T} 2$ was the order.

\section{$M C H C(g / d l)$}

In case of $\mathrm{T} 1$ mean was $36.18 \pm 1.97$ and range was 34.56-39.21 and in case of T2 mean and range were $24.87 \pm 1.56$ and $22.21-26.44$ respectively. In T3 mean was $56.84 \pm 3.38$ and range was $51.66-60.98$. The arrangement was in an order T3>T1>T2. 
Table 1: Hematological profile of Labeo calbasu under three different treatments with 15\%, 20\% and 25\% dietary (Crude) protein levels respectively.

\begin{tabular}{|c|c|c|c|c|c|c|}
\hline \multirow{3}{*}{$\begin{array}{l}\text { Haematological } \\
\text { variables }\end{array}$} & \multicolumn{6}{|c|}{ Diet variables } \\
\hline & \multicolumn{2}{|c|}{ First $(15 \% \mathrm{CP})$} & \multicolumn{2}{|c|}{ Second $(20 \% \mathrm{CP})$} & \multicolumn{2}{|c|}{ Third (25\%CP) } \\
\hline & $\begin{array}{l}\text { Average values with } \\
\text { standard deviation }\end{array}$ & Value range & $\begin{array}{l}\text { Average values with } \\
\text { standard deviation }\end{array}$ & Value range & $\begin{array}{l}\text { Average values with } \\
\text { standard deviation }\end{array}$ & Value range \\
\hline $\mathrm{WBC}\left(10^{3} / \mu \mathrm{L}\right)$ & $78.74 \pm 6.82$ & $67.5-87.2$ & $84.58 \pm 5.55$ & $77.5-92.4$ & $75.58 \pm 4.43$ & $68.9-81.2$ \\
\hline LYM $\left(10^{3} / \mu \mathrm{L}\right)$ & $72.56 \pm 6.29$ & $62.5-81.1$ & $76.88 \pm 5.56$ & $69.6-84.3$ & $69.76 \pm 4.32$ & $63.4-74.6$ \\
\hline $\operatorname{MON}\left(10^{3} / \mu \mathrm{L}\right)$ & $4.44 \pm 0.47$ & $3.6-4.9$ & $5.6 \pm 0.72$ & $4.3-6.4$ & $4.1 \pm 0.4$ & $3.5-4.7$ \\
\hline $\operatorname{GRA}\left(10^{3} / \mu \mathrm{L}\right)$ & $1.74 \pm 0.53$ & $1.2-2.7$ & $2.1 \pm 0.87$ & $1.0-3.6$ & $1.72 \pm 0.81$ & $0.9-2.7$ \\
\hline LYM (\%) & $92.16 \pm 0.69$ & $91.03-93.00$ & $90.86 \pm 0.94$ & $89.81-92.42$ & $92.29 \pm 1.34$ & $90.33-94.42$ \\
\hline MON (\%) & $5.64 \pm 0.31$ & $5.28-6.14$ & $6.62 \pm 0.77$ & $5.55-7.7$ & $5.46 \pm 0.72$ & $4.44-6.24$ \\
\hline GRA (\%) & $2.21 \pm 0.61$ & $1.50-3.23$ & $2.52 \pm 1.16$ & $1.17-4.65$ & $2.25 \pm 0.98$ & $1.14-3.53$ \\
\hline $\operatorname{RBC}\left(10^{6} / \mu \mathrm{L}\right)$ & $1.43 \pm 0.19$ & $1.13-1.72$ & $1.85 \pm 0.16$ & $1.65-2.05$ & $1.17 \pm 0.11$ & $0.99-1.28$ \\
\hline HGB (g/dl) & $7.74 \pm 0.30$ & $7.3-8.2$ & $7.66 \pm 0.16$ & $7.5-7.9$ & $8.16 \pm 0.31$ & $7.6-8.5$ \\
\hline $\operatorname{HCT}(\%)$ & $31.58 \pm 2.54$ & $28.6-35.5$ & $34.16 \pm 0.96$ & $32.6-35.3$ & $37.32 \pm 1.64$ & $34.6-39.4$ \\
\hline $\operatorname{MCV}\left(\mu \mathrm{m}^{3}\right)$ & $161.52 \pm 16.10$ & $133.4-182.7$ & $167.92 \pm 8.09$ & $158.0-176.8$ & $123.94 \pm 6.28$ & $112.7-131.2$ \\
\hline $\mathrm{MCH}(\mathrm{pg})$ & $58.32 \pm 5.78$ & $52.3-69.2$ & $41.79 \pm 3.66$ & $38.05-46.75$ & $70.44 \pm 5.42$ & $63.28-76.77$ \\
\hline MCHC (g/dl) & $36.18 \pm 1.97$ & $34.56-39.21$ & $24.87 \pm 1.56$ & $22.21-26.44$ & $56.84 \pm 3.38$ & $51.66-60.98$ \\
\hline RDW (\%) & $8.06 \pm 0.15$ & $7.8-8.2$ & $7.26 \pm 0.33$ & $6.8-7.7$ & $8.94 \pm 0.38$ & $8.3-9.4$ \\
\hline RDW-SD $\left(\mu \mathrm{m}^{3}\right)$ & $77.1 \pm 1.93$ & $74.5-79.3$ & $65.88 \pm 3.91$ & $61.8-71.5$ & $81.68 \pm 2.38$ & $77.8-85.2$ \\
\hline $\operatorname{PLT}\left(10^{3} / \mu \mathrm{L}\right)$ & $69.28 \pm 7.92$ & $58.3-81.4$ & $57.34 \pm 5.64$ & $47.8-64.1$ & $69.04 \pm 5.2$ & $61.3-76.1$ \\
\hline $\operatorname{MPV}\left(\mu \mathrm{m}^{3}\right)$ & $7.42 \pm 0.6$ & $6.5-8.2$ & $6.98 \pm 0.35$ & $6.4-7.5$ & $7.8 \pm 0.72$ & $6.9-8.8$ \\
\hline PCT (\%) & $0.058 \pm 0.018$ & $0.038-0.086$ & $0.048 \pm 0.021$ & $0.021-0.076$ & $0.057 \pm 0.019$ & $0.036-0.084$ \\
\hline PDW (\%) & $7.96 \pm 0.4$ & $7.3-8.5$ & $7.66 \pm 0.44$ & $7.0-8.2$ & $7.74 \pm 0.34$ & $7.2-8.2$ \\
\hline
\end{tabular}

\section{$R D W(\%)$}

In case of T1 mean was $8.06 \pm 0.15$ and range was 7.8 8.2. In T2 mean was $7.26 \pm 0.33$ and range was $6.8-$ 7.7. In T3 mean was $8.94 \pm 0.38$ and range was 8.3 9.4. $\mathrm{T} 3>\mathrm{T} 1>\mathrm{T} 2$ was the sequence.

\section{$R D W-S D\left(\mu m^{3}\right)$}

In case of T1 mean was $77.1 \pm 1.93$ and range was $74.5-$ 79.3. In T2 mean was $65.88 \pm 3.91$ and range was $61.8-$ 71.5. In T3 mean and range were $81.68 \pm 2.38$ and 77.885.2 respectively. The arrangement could be $\mathrm{T} 3>\mathrm{T} 1>\mathrm{T} 2$.

\section{$\operatorname{PLT}\left(10^{3} / \mu \mathrm{L}\right)$}

In T1 mean was 69.28 \pm 7.92 and range was 58.3-81.4. In T2 mean was $57.34 \pm 5.64$ and range was $47.8-64.1$ and in case of T3 mean was $69.04 \pm 5.2$ and range was 61.3-76.1. T1>T3>T2.

\section{$\operatorname{MPV}\left(\mu \mathrm{m}^{3}\right)$}

In case of $\mathrm{T} 1$ mean was $7.42 \pm 0.6$ and range was 6.5 8.2. In T2 mean was $6.98 \pm 0.35$ and range was $6.4-7.5$ and in case of T3 mean was $7.8 \pm 0.72$ and range was 6.9-8.8. T3>T1 $>\mathrm{T} 2$ was the arrangement.

\section{PCT (\%)}

In $\mathrm{T} 1$ mean was $0.058 \pm 0.018$ and range was 0.038 0.086 . In $\mathrm{T} 2$ mean was $0.048 \pm 0.021$ and range was $0.021-0.076$. In T3 mean was $0.057 \pm 0.019$ and range was $0.036-0.084$. T1 $>$ T $3>\mathrm{T} 2$.

\section{PDW (\%)}

In T1 mean was $7.96 \pm 0.4$ and range was $7.3-8.5$. In T2 mean was $7.66 \pm 0.44$ and range was $7.0-8.2$. In case of T3 mean was $7.74 \pm 0.34$ and range was 7.2 8.2. $\mathrm{T} 1>\mathrm{T} 3>\mathrm{T} 2$ was the sequence.

Table 2 described statistics and regression results of different blood parameters with total length (TL) of Labeo calbasu under three treatments as 15\%,20\% and $25 \%$ dietary protein levels respectively.

\section{Wet body weight and total length}

Studies of the relationship between body weight in wet and length (total) confirmed highly significant $(\mathrm{p}<0.001)$ correlation when analyzed value of " $\mathrm{r}$ " in three feeds ( 0.962 in case of T1, 0.998 in case of T2, 0.989 in case of T3). 
Table 2: Descriptive statistics and regression results of some blood parameters with respect to fish body total length (TL) (cm) of Labeo calbasu cultured under different crude protein levels.

\begin{tabular}{|c|c|c|c|c|c|c|c|c|}
\hline \multirow[t]{2}{*}{ Equation } & \multirow{2}{*}{$\begin{array}{l}\text { Diet } \\
\text { variables }\end{array}$} & \multicolumn{2}{|c|}{ Regression results } & \multirow{2}{*}{$\begin{array}{l}95 \text { percent } \mathrm{CI} \text { in } \\
\text { case of a }\end{array}$} & \multirow{2}{*}{$\begin{array}{l}95 \text { percent } \mathrm{CI} \text { in } \\
\text { case of } b\end{array}$} & \multirow{2}{*}{$\begin{array}{l}\text { SE (standard } \\
\text { error) }\end{array}$} & \multicolumn{2}{|c|}{ Value of Value of } \\
\hline & & a & $\mathbf{b}$ & & & & & \\
\hline \multirow[t]{3}{*}{$\mathrm{W}=\mathrm{a}+\mathrm{b} \mathrm{TL}$} & $\mathrm{T} 1(15 \%)$ & -1.74 & 2.83 & $-3.155-0.319$ & $1.251-4.301$ & 0.46 & 0.962 & 0.925 \\
\hline & $\mathrm{T} 2(20 \%)$ & -1.758 & 2.89 & $-2.111--1.405$ & $2.555-3.228$ & 0.11 & 0.998 & 0.996 \\
\hline & $\mathrm{T} 3(25 \%)$ & -1.396 & 2.55 & $-2.127--0.666$ & $1.851-3.250$ & 0.22 & 0.989 & 0.978 \\
\hline \multirow[t]{3}{*}{$\mathrm{K}=\mathrm{a}+\mathrm{b} \mathrm{TL}$} & $\mathrm{T} 1(15 \%)$ & 1.479 & -0.025 & $-0.365-3.324$ & $-0.226-0.176$ & 0.06 & 0.224 & 0.05 \\
\hline & $\mathrm{T} 2(20 \%)$ & 1.481 & -0.012 & $1.051-1.911$ & $-0.050-0.026$ & 0.01 & 0.510 & 0.260 \\
\hline & T3(25\%) & 1.960 & -0.053 & $0.956-2.964$ & $-0.141-0.036$ & 0.03 & 0.737 & 0.544 \\
\hline \multirow[t]{3}{*}{$P L T=a+b$ TL } & $\mathrm{T} 1(15 \%)$ & 0.608 & 1.280 & $-0.645-1.862$ & $-0.024-2.584$ & 0.41 & 0.875 & 0.765 \\
\hline & $\mathrm{T} 2(20 \%)$ & 1.919 & -0.156 & $0.487-3.352$ & $-1.523-1.211$ & 0.43 & 0.205 & 0.042 \\
\hline & T3(25\%) & 1.908 & -0.068 & $1.029-2.788$ & $-0.910-0.775$ & 0.26 & 0.146 & 0.021 \\
\hline \multirow[t]{3}{*}{$W B C s=a+b T L$} & $\mathrm{~T} 1(15 \%)$ & 1.452 & 0.461 & $-0.390-3.293$ & $-1.455-2.377$ & 0.6 & 0.404 & 0.163 \\
\hline & $\mathrm{T} 2(20 \%)$ & 2.069 & -0.136 & $1.161-2.977$ & $-1.003-0.731$ & 0.27 & 0.277 & 0.077 \\
\hline & $\mathrm{T} 3(25 \%)$ & 1.860 & 0.017 & $1.167-2.552$ & $-0.646-0.680$ & 0.21 & 0.048 & 0.002 \\
\hline \multirow[t]{3}{*}{$\mathrm{RBC} s=\mathrm{a}+\mathrm{b} \mathrm{TL}$} & $\mathrm{T} 1(15 \%)$ & 0.841 & -0.717 & $-2.022-3.705$ & $-3.696-2.263$ & 0.94 & 0.404 & 0.163 \\
\hline & $\mathrm{T} 2(20 \%)$ & 0.826 & -0.536 & $0.184-1.467$ & $-1.149-0.076$ & 0.19 & 0.849 & 0.721 \\
\hline & T3(25\%) & -0.186 & 0.241 & $-1.175-0.803$ & $-0.706-1.188$ & 0.30 & 0.423 & 0.179 \\
\hline \multirow[t]{3}{*}{$\mathrm{HGB}=\mathrm{a}+\mathrm{b} \mathrm{TL}$} & $\mathrm{T} 1(15 \%)$ & 1.136 & -0.258 & $0.384-1.888$ & $-1.041-0.525$ & 0.25 & 0.518 & 0.268 \\
\hline & T2(20\%) & 0.927 & -0.041 & $0.633-1.220$ & $-0.321-0.239$ & 0.09 & 0.258 & 0.067 \\
\hline & T3(25\%) & 0.957 & -0.043 & $0.515-1.398$ & $-0.466-0.379$ & 0.13 & 0.186 & 0.035 \\
\hline \multirow[t]{3}{*}{$L Y M=a+b T L$} & $\mathrm{~T} 1(15 \%)$ & 1.398 & 0.480 & $-0.417-3.213$ & $-1.409-2.368$ & 0.59 & 0.423 & 0.179 \\
\hline & T2(20\%) & 2.051 & -0.159 & $1.050-3.052$ & $-1.115-0.797$ & 0.3 & 0.292 & 0.085 \\
\hline & T3(25\%) & 1.853 & -0.01 & $1.123-2.582$ & $-0.708-0.689$ & 0.22 & 0.026 & 0.0006 \\
\hline \multirow[t]{3}{*}{$\mathrm{MON}=\mathrm{a}+\mathrm{b} \mathrm{TL}$} & $\mathrm{T} 1(15 \%)$ & 0.763 & -0.123 & $-1.748-3.274$ & $-2.736-2.489$ & 0.82 & 0.086 & 0.007 \\
\hline & $\mathrm{T} 2(20 \%)$ & 0.362 & 0.365 & $-1.493-2.217$ & $-1.406-2.136$ & 0.56 & 0.354 & 0.125 \\
\hline & T3(25\%) & 0.337 & 0.262 & $-0.698-1.372$ & $-0.729-1.254$ & 0.31 & 0.438 & 0.191 \\
\hline \multirow[t]{3}{*}{$\mathrm{GRA}=\mathrm{a}+\mathrm{b} \mathrm{TL}$} & $\mathrm{T} 1(15 \%)$ & -0.976 & 1.248 & $-6.988-5.035$ & $-5.007-7.502$ & 1.96 & 0.344 & 0.118 \\
\hline & T2(20\%) & 0.536 & -0.241 & $-5.511-6.584$ & $-6.014-5.532$ & 1.814 & 0.076 & 0.006 \\
\hline & $\mathrm{T} 3(25 \%)$ & -0.255 & 0.425 & $-5.671-5.161$ & $-4.762-5.611$ & 1.63 & 0.149 & 0.022 \\
\hline \multirow[t]{3}{*}{$M C V=a+b T L$} & $\mathrm{~T} 1(15 \%)$ & 1.998 & 0.217 & $-0.320-4.315$ & $-2.195-2.629$ & 0.76 & 0.163 & 0.027 \\
\hline & T2(20\%) & 1.997 & 0.218 & $1.438-2.556$ & $-0.316-0.751$ & 0.17 & 0.600 & 0.360 \\
\hline & T3(25\%) & 2.399 & -0.294 & $2.178-2.620$ & $-0.506-0.083$ & 0.07 & 0.931 & 0.867 \\
\hline \multirow[t]{3}{*}{$\mathrm{MCH}=\mathrm{a}+\mathrm{b}$ TL } & $\mathrm{T} 1(15 \%)$ & 1.104 & 0.686 & $-0.669-2.878$ & $-1.158-2.532$ & 0.58 & 0.564 & 0.318 \\
\hline & T2(20\%) & 1.101 & 0.495 & $0.292-1.910$ & $-0.277-1.268$ & 0.24 & 0.763 & 0.582 \\
\hline & T3(25\%) & 2.142 & -0.284 & $1.425-2.860$ & $-0.971-0.403$ & 0.22 & 0.605 & 0.366 \\
\hline \multirow[t]{3}{*}{$\mathrm{MCHC}=a+b$ TL } & $\mathrm{T} 1(15 \%)$ & 1.107 & 0.469 & $0.214-1.999$ & $-0.459-1.398$ & 0.29 & 0.681 & 0.463 \\
\hline & T2(20\%) & 1.104 & 0.278 & $0.348-1.860$ & $-0.444-0.999$ & 0.23 & 0.577 & 0.333 \\
\hline & $\mathrm{T} 3(25 \%)$ & 1.743 & 0.01 & $1.038-2.448$ & $-0.665-0.685$ & 0.21 & 0.027 & 0.0007 \\
\hline \multirow[t]{3}{*}{ RDW-SD $=a+b T L$} & $\mathrm{~T} 1(15 \%)$ & 2.06 & -0.18 & $1.586-2.533$ & $-0.672-0.313$ & 0.15 & 0.557 & 0.310 \\
\hline & T2(20\%) & 1.483 & 0.320 & $0.906-2.060$ & $-0.230-0.871$ & 0.17 & 0.730 & 0.533 \\
\hline & T3(25\%) & 1.960 & -0.046 & $1.630-2.291$ & $-0.363-0.270$ & 0.1 & 0.261 & 0.068 \\
\hline \multirow[t]{3}{*}{$M P V=a+b T L$} & $\mathrm{~T} 1(15 \%)$ & 1.725 & -0.892 & $0.754-2.697$ & $-1.902-0.119$ & 0.32 & 0.851 & 0.724 \\
\hline & T2(20\%) & 0.886 & -0.041 & $0.154-1.619$ & $-0.740-0.658$ & 0.22 & 0.108 & 0.012 \\
\hline & Т3(25\%) & 0.879 & 0.011 & $-0.199-1.956$ & $-1.020-1.043$ & 0.32 & 0.020 & 0.0004 \\
\hline
\end{tabular}

Correlation coefficient designated as $r$, coefficient of determination designated as $r^{2}$, intercept designated as a, regression coefficient designated as b. Confidence intervals designated as $\mathrm{Cl}$, standard error designated as S.E. 
Table 3: Descriptive statistics and regression results of some blood parameters with respect to fish weight $(W)(g)$ of Labeo calbasu cultured by using different crude protein levels.

\begin{tabular}{|c|c|c|c|c|c|c|c|c|}
\hline \multirow[t]{2}{*}{ Equation } & \multirow{2}{*}{$\begin{array}{l}\text { Diet Varia- } \\
\text { bles }\end{array}$} & \multicolumn{2}{|c|}{ Regression results } & \multirow{2}{*}{$\begin{array}{l}\text { 95percent CI of in } \\
\text { case of a }\end{array}$} & \multirow{2}{*}{$\begin{array}{l}\text { 95percent } \mathrm{CI} \text { in } \\
\text { case of } b\end{array}$} & \multirow{2}{*}{$\begin{array}{l}\text { SE or stand- } \\
\text { ard error }\end{array}$} & \multicolumn{2}{|c|}{ Value of Value } \\
\hline & & a & b & & & & & of " $r$ " \\
\hline \multirow{3}{*}{$\mathrm{CF}=\mathrm{a}+\mathrm{b} \mathrm{W}$} & $\mathrm{T} 1(15 \%)$ & 1.239 & 0.001 & $0.533-1.945$ & $-0.07-0.072$ & 0.02 & 0.029 & 0.0098 \\
\hline & T2(20\%) & 1.384 & -0.002 & $1.235-1.532$ & $-0.009-0.005$ & 0.002 & 0.479 & 0.229 \\
\hline & T3(25\%) & 1.535 & -0.008 & $1.052-2.018$ & $-0.03-0.014$ & 0.007 & 0.565 & 0.319 \\
\hline \multirow{3}{*}{$\mathrm{PLT}=\mathrm{a}+\mathrm{b} \mathrm{W}$} & $\mathrm{T} 1(15 \%)$ & 1.463 & 0.384 & $0.889-2.036$ & $-0.199-0.967$ & 0.18 & 0.770 & 0.593 \\
\hline & $\mathrm{T} 2(20 \%)$ & 1.809 & -0.041 & $1.201-2.417$ & $-0.517-0.435$ & 0.15 & 0.158 & 0.025 \\
\hline & $\mathrm{T} 3(25 \%)$ & 1.858 & -0.016 & $1.438-2.276$ & $-0.344-0.313$ & 0.1 & 0.087 & 0.007 \\
\hline \multirow[t]{3}{*}{$W B C s=a+b W$} & $\mathrm{~T} 1(15 \%)$ & 1.759 & 0.138 & $1.105-2.414$ & $-0.528-0.804$ & 0.21 & 0.356 & 0.127 \\
\hline & $\mathrm{T} 2(20 \%)$ & 1.991 & -0.051 & $1.611-2.370$ & $-0.348-0.246$ & 0.09 & 0.300 & 0.090 \\
\hline & T3(25\%) & 1.855 & 0.018 & $1.530-2.180$ & $-0.237-0.273$ & 0.08 & 0.128 & 0.016 \\
\hline \multirow[t]{3}{*}{$\mathrm{RBCs}=\mathrm{a}+\mathrm{b} \mathrm{W}$} & $\mathrm{T} 1(15 \%)$ & 0.286 & -0.137 & $-0.775-1.348$ & $-1.217-0.943$ & 0.34 & 0.227 & 0.052 \\
\hline & $\mathrm{T} 2(20 \%)$ & 0.497 & -0.183 & $0.220-0.773$ & $-0.4-0.033$ & 0.07 & 0.841 & 0.708 \\
\hline & T3(25\%) & -0.048 & 0.09 & $-0.520-0.423$ & $-0.280-0.460$ & 0.12 & 0.408 & 0.166 \\
\hline \multirow[t]{3}{*}{$\mathrm{HGB}=\mathrm{a}+\mathrm{b} \mathrm{W}$} & $\mathrm{T} 1(15 \%)$ & 0.987 & -0.100 & $0.740-1.233$ & $-0.351-0.151$ & 0.08 & 0.592 & 0.350 \\
\hline & $\mathrm{T} 2(20 \%)$ & 0.902 & -0.014 & $0.779-1.026$ & $-0.111-0.083$ & 0.03 & 0.260 & 0.067 \\
\hline & $\mathrm{T} 3(25 \%)$ & 0.923 & -0.009 & $0.712-1.134$ & $-0.175-0.157$ & 0.05 & 0.102 & 0.01 \\
\hline \multirow[t]{3}{*}{$L Y M=a+b W$} & $\mathrm{~T} 1(15 \%)$ & 1.726 & 0.136 & $1.074-2.378$ & $-0.528-0.780$ & 0.21 & 0.352 & 0.124 \\
\hline & T2(20\%) & 1.958 & -0.058 & $1.539-2.377$ & $-0.386-0.270$ & 0.1 & 0.309 & 0.095 \\
\hline & T3(25\%) & 1.837 & 0.004 & $1.492-2.182$ & $-0.267-0.275$ & 0.08 & 0.029 & 0.0008 \\
\hline \multirow[t]{3}{*}{$\mathrm{MON}=\mathrm{a}+\mathrm{b} \mathrm{W}$} & $\mathrm{T} 1(15 \%)$ & 0.688 & -0.045 & $-0.185-1.562$ & $-0.933-0.844$ & 0.28 & 0.092 & 0.008 \\
\hline & $\mathrm{T} 2(20 \%)$ & 0.597 & 0.116 & $-0.192-1.386$ & $-0.502-0.734$ & 0.19 & 0.326 & 0.106 \\
\hline & T3(25\%) & 0.469 & 0.112 & $-0.008-0.946$ & $-0.262-0.487$ & 0.12 & 0.483 & 0.233 \\
\hline \multirow[t]{3}{*}{$G R A=a+b W$} & $\mathrm{~T} 1(15 \%)$ & -0.471 & 0.709 & $-2.295-1.353$ & $-1.147-2.565$ & 0.58 & 0.574 & 0.330 \\
\hline & T2(20\%) & 0.426 & -0.112 & $-2.112-2.965$ & $-2.1-1.876$ & 0.62 & 0.103 & 0.011 \\
\hline & T3(25\%) & -0.204 & 0.310 & $-2.691-2.283$ & $-1.642-2.263$ & 0.61 & 0.280 & 0.079 \\
\hline \multirow[t]{3}{*}{$M C V=a+b W$} & $\mathrm{~T} 1(15 \%)$ & 2.240 & -0.035 & $1.425-3.056$ & $-0.865-0.794$ & 0.26 & 0.078 & 0.006 \\
\hline & T2(20\%) & 2.132 & 0.073 & $1.893-2.371$ & $-0.114-0.260$ & 0.06 & 0.583 & 0.339 \\
\hline & T3(25\%) & 2.239 & -0.116 & $2.147-2.331$ & $-0.188--0.044$ & 0.02 & 0.947 & 0.897 \\
\hline \multirow[t]{3}{*}{$\mathrm{MCH}=\mathrm{a}+\mathrm{b} \mathrm{W}$} & $\mathrm{T} 1(15 \%)$ & 1.626 & 0.14 & $0.923-2.330$ & $-0.575-0.856$ & 0.22 & 0.339 & 0.115 \\
\hline & T2(20\%) & 1.405 & 0.169 & $1.060-1.751$ & $-0.101-0.440$ & 0.08 & 0.754 & 0.569 \\
\hline & T3(25\%) & 1.971 & -0.099 & $1.614-2.329$ & $-0.380-0.181$ & 0.09 & 0.544 & 0.297 \\
\hline \multirow[t]{3}{*}{$\mathrm{MCHC}=\mathrm{a}+\mathrm{b} \mathrm{W}$} & $\mathrm{T} 1(15 \%)$ & 1.386 & 0.175 & $1.105-1.667$ & $-0.111-0.462$ & 0.09 & 0.748 & 0.559 \\
\hline & T2(20\%) & 1.273 & 0.096 & $0.955-1.591$ & $-0.153-0.345$ & 0.08 & 0.579 & 0.335 \\
\hline & T3(25\%) & 1.733 & 0.017 & $1.401-2.064$ & $-0.243-0.277$ & 0.08 & 0.119 & 0.014 \\
\hline \multirow[t]{3}{*}{$\mathrm{RDW}-\mathrm{SD}=\mathrm{a}+\mathrm{b} \mathrm{W}$} & $\mathrm{T} 1(15 \%)$ & 1.949 & -0.064 & $1.788-2.111$ & $-0.228-0.100$ & 0.05 & 0.581 & 0.338 \\
\hline & T2(20\%) & 1.681 & 0.108 & $1.433-1.929$ & $-0.086-0.303$ & 0.06 & 0.716 & 0.513 \\
\hline & T3(25\%) & 1.927 & -0.012 & $1.767-2.086$ & $-0.137-0.114$ & 0.04 & 0.168 & 0.028 \\
\hline \multirow[t]{3}{*}{$M P V=a+b W$} & $\mathrm{~T} 1(15 \%)$ & 1.145 & -0.282 & $0.751-1.538$ & $-0.683-0.118$ & 0.13 & 0.792 & 0.626 \\
\hline & T2(20\%) & 0.858 & -0.012 & $0.550-1.167$ & $-0.254-0.229$ & 0.07 & 0.091 & 0.008 \\
\hline & Т3(25\%) & 0.901 & -0.008 & $0.391-1.410$ & $-0.408-0.392$ & 0.13 & 0.038 & 0.001 \\
\hline
\end{tabular}

Correlation coefficient designated as $r$, coefficient of determination designated as $r^{2}$, intercept designates as a, regression coefficient designates as b. Confidence intervals designated as $\mathrm{Cl}$, standard error designated as S.E.

Condition factor and total length was found to be non-significant (ns) in case of all the Relationship of condition factor with total length treatments with 15\%,20\% and $25 \%$ dietary protein June 2020 | Volume 36 | Issue 2 | Page 512 
level respectively $(p>0.05)$.

Relation between various blood parameters and total length

When correlation between different hematological parameters like platelets, White blood cells, Red blood cells, Haemoglobin, Lymphocytes, Monocytes, Granulocytes, MCV (Mean corpuscular volume), $\mathrm{MCH}$ (Mean corpuscular hemoglobin), MCHC (Mean corpuscular hemoglobin concentration), RDW-SD (Red blood cell distribution-standard deviation included), MPV (Mean platelets volume) and total length was studied it was found to be nonsignificant in case of all the feeding groups with $15 \%$, $20 \%$ and $25 \%$ dietary protein levels respectively $(\mathrm{p}>0.05)$.

Statistics and regression results of different blood indices with body weight (wet) in grams of Labeo calbasu reared under three different treatments ( $\mathrm{T} 1$, T2 and T3) with 15\%, 20\% and 25\% dietary protein levels respectively were recorded in Table 3 .

\section{Condition factor $(K)$ and body weight (Wet)}

The relationship between $\mathrm{K}$ and body weight was found to be non-significant in case of all the feeding groups ( $p>0.05)$.

Correlation between haematological indices and wet body weight $(W)$

When correlation between different hematological parameters like platelets, White blood cells, Red blood cells, Haemoglobin, Lymphocytes, Monocytes, Granulocytes, MCV (Mean corpuscular volume), $\mathrm{MCH}$ (mean corpuscular hemoglobin), MCHC (Mean corpuscular hemoglobin concentration), RDW-SD (Red blood cell distribution-standard deviation included), MPV (Mean platelets volume) and total length was studied it was found to be nonsignificant in case of all the feeding groups with $15 \%$, $20 \%$ and $25 \%$ dietary protein levels respectively $(\mathrm{p}>0.05)$.

The hematological profile is an imperative, successful and vulnerable checking apparatus to decide the physiological, neurotic and useful status (wellbeing) of a life form (Kohanestani et al., 2013). According to Southamani et al. (2015), hematological records have the farthest incentive in speaking to the deviation and aggravation in physiological states of fish, clinical analysis, and level of biological community defilements and impacts of dangerous substances. Varieties in hematological qualities, for the most part, relied upon a wide scope of nutritious and antagonistic to nutritious elements of the feed used for fish (Osuigwe et al., 2007). Blood profile showed significant variations when plant origin diet was added as a supplement to fish feed (Anyanwu et al., 2011). Oreochromis niloticus (Nile Tilapia) bolstered with a various additional range of maltose diet likewise showed varieties in blood attributes (Ighwela et al., 2012). According to El-Kasheif et al. (2011), a non-noteworthy relationship among different hematological lists was seen in Nile Tilapia (O. niloticus) bolstered with commercially prepared feed with fish oil added as supplement. In condition of the above research works, present examination was organized to set up typical range scope of hematological qualities of Labeo calbasu sustained with fluctuating dietary protein levels.

Iqbal and Naeem (2016) revealed that increase in the value of Red blood cells (RBC), Mean corpuscular volume (MCV), White blood cells (WBC), Hematocrit (HCT) and Lymphocytes (LYM) in Labeo robita fed with diet containing protein level greater than $25 \%$ indicated that fish health status became stressful. RBC, MCV, WBC, HCT and Lymphocytes if goes on increasing are indicators of the stressful health status of the fish. In their study, the fish fed with $25 \%$ dietary protein level showed the best health status and also showed an increasing trend in hematological indices like $\mathrm{MCH}$, Platelets, $\mathrm{MCHC}$ and PCT. The increase in the number of these indices are actually indicators of good health of the fish. They unraveled the fact in their study that fish fed with 25 percent protein in feed showed best health status in comparison to other higher percentages of proteins like 30\%, 35\% and 40\%. This was found exactly according to the present study with Labeo calbasu fed with $25 \%$ dietary protein level showed good health status of the fish as compared to other dietary protein levels like $15 \%$ and $20 \%$. The increase in RBC and WBC with increase in dietary protein level feed (the indicators of stress health status in the fish) as studied by Iqbal and Naeem (2016) and Nasir and Al-Sraji (2013) also supported in case of Cyprinus carpio but is contrary to the research work by Yue and Zhou (2008) with juvenile hybrid tilapia and Iqbal et al. (2016) with juvenile Labeo rohita.

In the present study, fish were fed with $15 \%, 20 \%$ June 2020 | Volume 36 | Issue 2 | Page 513 
and $25 \%$ dietary protein level. Fish with $25 \%$ dietary protein level showed good health status in terms of hematological parameters like $\mathrm{MCH}, \mathrm{MCHC}$, Platelets as compared to other dietary protein levels i.e., $15 \%$ and $20 \%$ dietary protein levels. The value of PCT (\%) also showing good value in $25 \%$ dietary protein level treatment was an indicator of the better health status of the fish. Fish fed with protein levels $30 \%, 35 \%$ and $40 \%$ showed stressful health status (Iqbal and Naeem, 2016).

Values of gained parameters by Iqbal and Naeem (2016) in their study of Labeo rohita fed with $25 \%$ dietary protein level were nearly according to the values of present study of Labeo calbasu. The range values of different blood parameters as studied by Iqbal and Naeem (2016) are 1.3-9 for Monocytes $\left(10^{3} / \mu \mathrm{L}\right), 0.9-12.4$ for Granulocytes $\left(10^{6} / \mu \mathrm{L}\right), 81.9$ 97.8 for Lymphocytes (\%), 1.3-7.6 for Monocytes (\%), 0.9-10.5 for Granulocytes (\%), 1.81-2.57 for Red Blood Cells $\left(10^{6} / \mu \mathrm{L}\right), 6.8-9.6$ Hemoglobin (g/dl), 15.9-48.4 for Hematocrit (\%), 87.8-188.3 for Mean Corpuscular volume $\left(\mu \mathrm{m}^{3}\right)$, 9.5-16.3 for RDW (\%), 44.1-196.7 for RDW-SD $\left(\mu \mathrm{m}^{3}\right)$, 60.0-255.0 for PLT $\left(10^{3} / \mu \mathrm{L}\right), 6.2-7.8$ for MPV $\left(\mu \mathrm{m}^{3}\right), 0.04-0.18$ for PCT (\%), 7.1-9.8 for PDW (\%). The results for the range of the blood parameters in the present study as given in the table 1 are present within the limits of the range values of the above study by Iqbal and Naeem (2016).

The values in the present study for WBC $\left(10^{3} / \mu \mathrm{L}\right)$ in T1 (15\% dietary protein level), T2 (20\% dietary protein level) and T3 (25\% dietary protein level) were 67.5-87.2, 77.5-92.4 and 68.9-81.2 respectively were matched with the reference values described by Hrubec et al. (2000) for cultured tilapia i.e., 21.56154.69. The Values for Red Blood Cells (RBC 10\% $\mu \mathrm{L})$ in the present study were $1.13-1.72,1.65-2.05$ and $0.99-1.28$ for $\mathrm{T} 1, \mathrm{~T} 2$ and $\mathrm{T} 3$ respectively are closed to values described by Hrubec et al. (2000) as 1.91-2.88. The values for other hematological indices like Hemoglobin (g/dl) and Mean Corpuscular Volume $\left(\mu \mathrm{m}^{3}\right)$ as described by Hrubec et al. (2000) were 7-9.8 and 115-183 respectively were closed to values described in Table 1 in the present study. The values for Hemoglobin (g/dl) and Mean Corpuscular Volume $\left(\mu \mathrm{m}^{3}\right)$ for T1 with $15 \%$ dietary protein level in the present study were 7.3-8.2 and 133.4-182.7 respectively while the values for Hemoglobin $(\mathrm{g} / \mathrm{dl}$ ) and Mean Corpuscular Volume $\left(\mu \mathrm{m}^{3}\right)$ for T2 with 20\% dietary protein level were 7.5-7.9 and 158.0-
176.8 respectively. In T3 with a $25 \%$ dietary protein level, the values for Hemoglobin $(\mathrm{g} / \mathrm{dl})$ and Mean Corpuscular Volume $\left(\mu \mathrm{m}^{3}\right)$ were 7.6-8.5 and 112.7131.2 respectively in the present work. These all results were found close to reference values described by Hrubec et al. (2000).

Developed mature erythrocytes (RBCs) have a red color containing iron as a focal component to convey oxygen to different tissues (Rehulka, 2002). Stress states of a living being generally estimated through erythrocyte and leukocytes tally and its files esteem as these are dependable parameters. The higher values of WBC and RBC showed that the fish is in stressful health conditions. In the present investigation, $\mathrm{RBCs}$ and WBCs tally stray essentially on differing dietary protein levels. This work concluded that fish fed with $25 \%$ dietary protein level showed relatively better hematological indices as compared to $15 \%$ and $20 \%$ dietary protein level treatment as described in Table 1. Thus, it can be said that present study was exactly according to study by Iqbal and Naeem (2016) and Nasir and Al-Siraji (2013). Welker et al. (2007) in their study on channel catfish and Hisano et al. (2007) in their research on Nile tilapia found the results contrary to the present study as they found no noticeable difference in hematological parameters when fed with different diet supplements. This may be due to change in environment, habitat, feed ingredients and pollution.

\section{Conclusions and Recommendations}

In comprehensive clarifications, the fish fed at $25 \%$ dietary protein level as compared to $15 \%$ and $25 \%$ dietary protein levels showed better health status as compared to the other two diets. An expansion in hematological parameters RBCs, WBCs, HCT, $\mathrm{MCV}$ showed upsetting health status while an increase in values of platelets, $\mathrm{MCH}, \mathrm{MCHC}$ and $\mathrm{PCT}$ in $25 \%$ protein level showed vitality proportion diet speaks to great wellbeing status of the fish. The statistical analysis results revealed a very peculiar fact that correlations between hematological indices and total length or wet weight were found to be non-significant $(P>0.05)$ in the case of all the three groups with different protein feeding levels. This may be due to different intrinsic and extrinsic factors like age, sex, species, season, feed, feeding habits, habitat, temperature, time of the year, and culturing conditions etc. 
Novelty Statement

Results of the present study will helpful for fish feed and aquaculture industry to improve the production of this carp species (Labeo calbasu) in Pakistan.

\section{Author's Contribution}

Saira Bano performed experiments, analyzed the data, and wrote the first draft of the manuscript. Muhammad Naeem designed the experimentation and supervised the research work. Samrah Masud helped in data analysis and writing of the manuscript.

\section{Conflict of interest}

The authors declare that they have no conflict of interest.

\section{References}

Adeshina,I.,B.O.Emikpe,A.Jenyo-Oni,E.K.Ajani and M.I. Abubakar. 2019. Haematology, plasma biochemistry and serum of table size African catfish, Clarias gariepinus, naturally infected with listeria species in Oyo State. Compa. Clin. Pathol. 29(1): 69-73. https://doi.org/10.1007/ s00580-019-03034-6

Anyanwu, D.C., A.B.I. Udedibie, D.I. Osuigwe and V.O. Ogwo. 2011. Haematological responses of hybrid of Heterobranchus bidorsalis and Clarias gariepinus fed dietary levels of Carica papaya leaf meal. World Rural Observ. 3: 9-12.

Burgos-Aceves, M.A., L. Lionetti and C. Faggio. 2019. Multidisciplinary haematology as prognostic device in environmental and xenobiotic stress-induced response in fish. Sci. Total Environ. 670: 1170-1183. https://doi. org/10.1016/j.scitotenv.2019.03.275

El-Kasheif, M.A., S.S. Amal and A. Seham. 2011. Effects of varying levels of fish oil on growth performance, body composition and haematological characteristics of Nile tilapia (Oreochromis niloticus L). Egypt. J. Aquat. Biol. Fish. 15: 125-141. https://doi.org/10.21608/ ejabf.2011.2082

Ferdous, F. and T.R. Scott. 2015. A comparative examination of thrombocyte/platelet immunity. Immunol. Lett. 163(1): 32-39. https://doi. org/10.1016/j.imlet.2014.11.010

Hisano, H., M.M. Barros and L.E. Pezzato. 2007. Levedura e zincocomopró-nutrient esemraçõ espara tilápia do Nilo (Oreochromis niloticus: aspect shematológicos. Bol. Inst. Pesca. 33(1): 35-42. https://doi.org/10.1590/S0100204X2007000700017

Hrubec, T.C., J.L. Cardinale and S.A. Smith. 2000. Haematology and plasma chemistry reference intervals for cultured tilapia (Oreochromis hybrid). Vet. Clin. Pathol. 29(1): 7-12. https:// doi.org/10.1111/j.1939-165X.2000.tb00389.x

Ighwela, K.A, A.Z. Ahmad and A.B. Abol-Munafi. 2012. Haematological changes in Nile tilapia (Oreochromis niloticus fed with varying dietary maltose levels. World J. Fish. Mar. Sci. 4: 376381.

Iqbal, M.J. and M. Naeem. 2016. Haematological indices study of juvenile Labeo robita (Hamilton-1822) fed at varying protein: Energy ratios. Int. J. Fish Aquat. Stud. 4(5): 632-641.

Khan, I.A. and A. Maqbool. 2017. Effects of dietary protein levels on the growth, feed utilization and haemato-biochemical parameters of freshwater fish. Cyprinus Carpio Var. Specularis. Fish Aquacult. J. 8(1): 1-12.

Kohanestani, Z.M., A. Hajimoradloo, R. Ghorbani, S. Yulghi, A. Hoseini and M. Molaee. 2013. Seasonal variations in hematological parameters of alburnoides eichwaldii in zaringol streamGolestan province, Iran. World J. Fish Mar. Sci. 5(2): 121-126.

Moradi, N., M. Imanpoor and V. Taghizadeh. 2013. Hematological and biochemical changes induced by replacing fish meal with plant protein in the Cyprinus carpio Linnaeus (1785). Glob. Vet. 11(2): 233-237.

Naeem, M.A. Salam, S.S. Tahir and N. Rauf. 2010. Assessment of the essential element and toxic heavy metals in hatchery reared Oncorbynchus mykiss. Int. J. Agric. Biol. 12: 935-938.

Najim, S.M., S.S. Al-Noor and B.M Jasim. 2014. Effects of fish meal replacement with fish biosilage on some haematological and biochemical parameters in common carp Cyprinus carpio fingerlings. Int. J. Res. Fish Aquacult. 4(3): 112-116.

Nasir, N.A. and A.Y.J. Al-Sraji. 2013. Effect of Different dietary protein and fats on some biochemical blood parameters in common carp fingerlings (Cyprinus carpio L.) reared in float cages. Asian J. Exp. Biol. Sci. 4: 293-296.

Osuigwe, D.I.C. Nwosu and J.O. Ogunji. 2007. Preliminary observations on some 
haematological parameters of juvenile Heterobranchus longifilis fed different dietary levels of raw and boiled jackbean (Canavalia ensiformis) seed meal. Proceedings of Conference of International Agricultural Research Development, Tropentag, University of KasselWitzenhausen and University of Göttingen, October. pp. 9-11.

Rehulka, J., 2002. Aeromonas causes severe skin lesions in rainbow trout (Oncorbynchus mykiss): Clinical pathology, haematology and biochemistry. Acta. Vet. Brno. 71: 351-360. https://doi.org/10.2754/avb200271030351

Southamani, C., G. Shanthi and M. Deivasigamani. 2015. Hematological response in three Indian major carps in relation to supplementary feeding. Int. J. Fish Aquacult. Stud. 3(2): 287294.

Welker, T.L., C. Lim, M. Yildirim-Aksoy, R. Shelby and P.H. Klesius. 2007. Immune response and resistance to stress and Edwards iellaictaluri, challenge in channel catfish, Ictalurus punctatus fed diets containing commercial whole-cell yeast or yeast subcomponents. J. World Aquacult. Soc. 38(1): 24-35. https://doi.org/10.1111/j.17497345.2006.00070.x

Yue, Y.R. and Q.C. Zhou. 2008. Effect of replacing soybean meal with cottonseed meal on growth, feed utilization, and hematological indices for juvenile hybrid tilapia Oreochromis niloticus. Aquaculture. 284: 185-189. https://doi. org/10.1016/j.aquaculture.2008.07.030 